\title{
Decisões de investimento e restrição financeira: um modelo dinâmico com abordagem Bayesiana ${ }^{\dagger}$
}

\author{
Aquiles Elie Guimarães Kalatzis* \\ Camila Fernanda Bassetto
}

\begin{abstract}
RESUMO - Esse trabalho analisa a presença de restrição financeira nas decisões de investimento de 564 firmas brasileiras no período de 1997-2006, classificadas por porte e utilizando um modelo econométrico com enfoque bayesiano. Distribuições a priori são assumidas para os parâmetros, classificando o modelo em efeito fixo e efeito aleatório. $\mathrm{O}$ critério de seleção de modelos pela densidade preditiva mostrou-se o modelo de efeito fixo o mais adequado para a análise das decisões de investimento da firma. Os resultados indicaram que as firmas de menor porte sofrem de restrição financeira em suas decisões de investimento.
\end{abstract}

Palavras-chave: Decisão de investimento. Restrição financeira. Inferência bayesiana.

\section{INTRODUÇÃO}

A identificação da presença de restrição financeira nas decisões de investimento da firma tem sido um tema de grande importância na literatura da teoria do investimento em função de sua forte relação com o desenvolvimento financeiro e crescimento econômico. Nos últimos anos a principal discussão sobre a decisão de investimento da firma refere-se ao efeito da restrição financeira sobre o investimento. Essa questão intensificou-se a partir do seminal estudo de Fazzari, Hubbard e Petersen (1988), cujo principal resultado mostrou que as firmas mais restritas financeiramente eram aquelas que pagavam menores taxas de pagamento de dividendos. Diversos estudos posteriores mostraram que a forma como as firmas são classificadas é fundamental para apontar a presença de restrição financeira. Segundo Kaplan e Zingales (1997) uma firma é considerada restrita financeiramente se os custos ou a disponibilidade de fundos externos impedem a firma de realizar novos empreendimentos que ela teria escolhido se recursos internos estivessem disponíveis. Para Bond e Reenen (2002),

† A pesquisa reportada neste trabalho tem o apoio da Fundação de Amparo à Pesquisa do Estado de São Paulo FAPESP.

* Professor associado do Departamento de Engenharia de Produção da Escola de Engenharia de São Carlos. Endereço eletrônico: aquiles@sc.usp.br.

** Doutoranda em Engenharia de Produção no Departamento de Engenharia de Produção da Escola de Engenharia de São Carlos. Endereço eletrônico: camilafb@sc.usp.br. 
uma firma é restrita financeiramente se um aumento inesperado na disponibilidade de seus recursos internos gerar um aumento nos gastos com investimentos, sem que este aumento proporcione qualquer tipo de informação sobre o potencial de lucratividade da firma.

Uma questão intensamente discutida em recentes estudos se deve à forma de classificação das firmas, de maneira que se possam controlar e isolar os efeitos originados das restrições financeiras. Nesse sentido, as firmas consideradas nesse estudo foram classificadas por porte. Com o objetivo de explicar o comportamento do investimento da firma, diferentes estudos têm introduzido variáveis de liquidez reconhecendo o papel dos fundos internos como determinantes do investimento. A introdução de variáveis de liquidez na equação do investimento teve como propósito testar a hipótese de assimetria de informação.

Este trabalho procura contribuir com a literatura do investimento utilizando um modelo econométrico dinâmico sob uma abordagem bayesiana para tentar identificar a presença de restrição financeira nas decisões de investimento de 564 firmas brasileiras no período de 1997-2006. A motivação para o modelo bayesiano deve-se à possibilidade de escolher o modelo com maior capacidade de predição, através do critério das preditivas ordenadas, além da possibilidade de emitir considerações probabilísticas, diferentemente da abordagem frequentista.

O artigo está organizado em seções, incluindo esta introdução. Na seção 2 alguns aspectos referentes às decisões de investimento da firma são apresentados. Na seção 3 descreve-se o modelo econométrico e os dados, juntamente com a modelagem para estimação dos parâmetros, e a seleção de modelos. Nas seções 4 e 5 são apresentados os principais resultados econômicos e a conclusão deste trabalho, respectivamente.

\section{RESTRIÇÃO FINANCEIRA E DECISÕES DE INVESTIMENTO}

Estudos sobre o comportamento do investimento têm se concentrado na identificação da restrição financeira sobre as decisões de investimento da firma. Uma questão amplamente discutida tenta controlar e isolar os efeitos que surgem da restrição financeira considerando diferentes critérios para classificar as firmas.

O estudo de Fazzari, Hubbard e Petersen - FHP (1988) teve um impacto significante e intensificou a questão sobre os critérios de classificação das firmas para identificar a restrição financeira, motivando a realização de diversos trabalhos. FHP (1988) classificaram as firmas de acordo com a política de pagamento de dividendos e encontraram que a sensibilidade do investimento ao fluxo de caixa foi maior para as firmas de baixo pagamento 
de dividendos. Da classificação das firmas por taxa de pagamento de dividendos os autores inferiram a existência de uma relação monotonicamente crescente entre a sensibilidade do investimento ao fluxo de caixa e o grau de restrição financeira. Para Kaplan e Zingales (1997) não haveria razão teórica para uma relação monotonicamente crescente entre a sensibilidade do investimento ao fluxo de caixa e o grau de restrição financeira da firma. Os autores utilizaram a mesma amostra de FHP (1988) e mostraram que a relação entre a sensibilidade do investimento e o fluxo de caixa poderia ser de outra natureza antes de problemas de liquidez.

Evidências de que as firmas priorizam fontes internas como principal instrumento de financiamento de investimento foram encontradas por Devereux e Schiantarelli (1990), examinando o comportamento de 720 firmas na Inglaterra. Os dados analisados das firmas, agrupadas por tamanho, idade e tipo de industria indicaram que 67\% do total de recursos destinados ao financiamento de novos empreendimentos originavam-se dos lucros retidos das firmas.

A investigação de restrição financeira por diferentes formas de agrupamento das firmas tem sido proposta. Fazzari et al. (1988), por exemplo, classificaram as firmas por política de dividendos; Devereux e Schiantarelli (1990) utilizaram tamanho, maturidade e crescimento da firma como critério para separá-las; Schaller (1993) classificou as empresas por maturidade e grau de concentração de propriedade; Gilchrist e Himmelberg (1995) listaram as firmas conforme o acesso ao mercado de títulos e sua classificação de risco; Hsiao e Tahmiscioglu (1997) agruparam as firmas por intensidade de capital; Kaplan e Zingales (1997) dividiram a firmas por seus desempenhos operacionais, entre outras. $O$ fundamento teórico dessas distintas formas de separar as firmas tem como argumento permitir a identificação de algum grau de assimetria de informação. Por exemplo, a divulgação de firmas em boas condições financeiras, divulgando sua estrutura de capital, proporcionaria informações que indicariam a capacidade da firma em cumprir seus compromissos, diferentemente de firmas em frágeis condições financeiras.

Kalatzis e Azzoni (2009) classificaram um grupo de firmas brasileiras de acordo com a intensidade de capital e mostraram que as firmas mais intensivas em capital eram mais restritas e não estavam sujeitas à crítica que a variável liquidez estaria atuando como uma proxy para futuras oportunidades de investimento. 
Considerando a discussão sobre a variável de liquidez nas decisões de investimento, este trabalho busca contribuir com a literatura utilizando um modelo bayesiano com dados longitudinais para identificar a presença de restrição financeira.

\section{DADOS E MODELO}

O banco de dados contém informações de 564 firmas brasileiras no período de 1997 - 2006 ${ }^{1}$. As firmas foram classificadas por porte seguindo os critérios do Banco Nacional do Desenvolvimento Econômico e Social $\left(\right.$ BNDES$\left.^{2}\right)$. Para explicar o comportamento do investimento e identificar a presença de restrições financeiras utilizou-se o seguinte modelo dinâmico:

$$
\left(\frac{I}{K}\right)_{i t}=\alpha_{i}+\omega_{t}+\gamma_{1 j}\left(\frac{I}{K}\right)_{i, t-1}+\gamma_{2 j}\left(\frac{I}{K}\right)_{i, t-1}^{2}+\beta_{1 j}\left(\frac{F C}{K}\right)_{i t}+\beta_{2 j}\left(\frac{V}{K}\right)_{i t}+\beta_{3 j}\left(\frac{F I N}{K}\right)_{i t}+\varepsilon_{i t}
$$

onde $i$ representa as firmas, $i=1, \ldots, 564$; $t$ é o ano, $t=1, \ldots, 10 ; j$ é o grupo, $j=1,2 ; \alpha_{i}$ é o efeito específico da firma; $\omega_{t}$ é o componente temporal; $K$ é o estoque de capital (imobilizado); I é o investimento da firma; FC é o fluxo de caixa, o qual considera o efeito de possíveis restrições de liquidez sobre o investimento, embora represente também o potencial de rentabilidade futura da firma; $V$ são as vendas, as quais consideram o papel da taxa de mudança esperada no nível de vendas ou de produção, FIN é o financiamento total da empresa, considerado devido à ideia que uma maior alavancagem pode elevar o valor da firma, e $\varepsilon_{i t}$ é o erro. A variável dependente defasada procura contemplar o aspecto dinâmico do comportamento do investimento e a variável quadrática reflete um comportamento nãolinear no processo de ajustamento do estoque de capital.

Para obter as estimativas dos parâmetros, o software Winbugs foi utilizado e a convergência foi verificada pelo índice proposto por Gelman e Rubin (1992). Os modelos de efeito fixo e aleatório são caracterizados conforme as distribuições a priori atribuídas aos efeitos específico e temporal. No modelo de efeito fixo são assumidas as seguintes distribuições a priori:

\footnotetext{
${ }^{1}$ Fonte: Gazeta Mercantil, Revista Exame Maiores e Melhores, obtido pela Fundação Instituto de Pesquisas Contábeis, Atuariais e Financeiras do Departamento de Contabilidade da Universidade de São Paulo.

${ }^{2}$ As firmas de grande porte foram as que apresentaram receita bruta anual maior que 60 milhões de reais.
} 


$$
\left\{\begin{array}{l}
\alpha_{i} \sim U\left(e_{1}, f_{1}\right) ; e_{1}, f_{1} \text { conhecidos, } i=1,2, \ldots, 564 ; \\
\omega_{t} \sim U\left(e_{2}, f_{2}\right) ; e_{2}, f_{2} \text { conhecidos, } t=1, \ldots, 10 ; \\
\beta_{l j} \sim N\left(u_{l j}, v_{l j}^{2}\right) ; u_{l j}, v_{l j} \text { conhecidos, } l=1,2,3 ; j=1,2 ; \\
\sigma^{2} \sim I G(c, d) ; c, d \text { conhecidos. }
\end{array}\right.
$$

(2)

Para o modelo de efeito aleatório, as prioris possuem uma estrutura hierárquica:

Primeiro estágio: $\left\{\begin{array}{l}\alpha_{i} \sim N\left(g_{1}, \sigma_{\alpha}^{2}\right) ; g_{1} \text { conhecido, } i=1,2, \ldots, 564 ; \\ \omega_{t} \sim N\left(g_{2}, \sigma_{\omega}^{2}\right) ; g_{2} \text { conhecido, } t=1, \ldots, 10 ; \\ \beta_{l j} \sim N\left(u_{l j}, v_{l j}^{2}\right) ; u_{l j}, v_{l j} \text { conhecidos, } l=1,2,3 ; j=1,2 ; \\ \sigma^{2} \sim I G\left(c_{1}, d_{1}\right) ; c_{1}, d_{1} \text { conhecidos. }\end{array}\right.$

(3)

Segundo estágio: $\left\{\begin{array}{l}\sigma_{\alpha}^{2} \sim I G\left(c_{2}, d_{2}\right) ; c_{2}, d_{2} \text { conhecidos; } \\ \sigma_{\omega}^{2} \sim I G\left(c_{3}, d_{3}\right) ; c_{3}, d_{3} \text { conhecidos. }\end{array}\right.$

(4)

Considerando as distribuições a priori independentes entre si e combinando-as com a função de verossimilhança, obtém-se a posteriori conjunta para a estimação dos parâmetros.

A verificação do modelo assumindo efeito fixo e efeito aleatório foi realizada por meio do critério das densidades preditivas ordenadas - $\mathrm{DPO}^{3}$. Os valores obtidos para o logaritmo da DPO a posteriori para os modelos de efeito fixo e efeito aleatório foram 5160.53 e 5121.10, respectivamente, sendo o modelo de efeito fixo preferível.

\section{RESULTADOS}

Nesta seção são apresentados os resultados econômicos. A Tabela 1 apresenta os valores médios e os desvios padrão dos principais indicadores financeiros para as firmas em cada grupo.

Nota-se que as firmas de grande porte apresentaram melhores indicadores financeiros. As firmas de grande porte investem mais de 60\% a mais que as de médio porte, tem rentabilidade $(L L / P L)$ substancialmente maior e vendem mais. Por outro lado, as firmas de médio porte apresentaram piores indicadores, indicando maior necessidade de tais firmas em manter uma capacidade de reserva de ativos. Os baixos valores de liquidez $(F C / K)$ e rentabilidade para tais firmas podem estar refletindo maior necessidade de caixa.

\footnotetext{
${ }^{3}$ Para mais informações sobre critérios de seleção de modelos, ver Gelfand e Dey (1993).
} 
TABELA 1 - CARACTERÍSTICAS FINANCEIRAS DAS FIRMAS - VALORES MÉDIOS E DESVIOS PADRÃO.

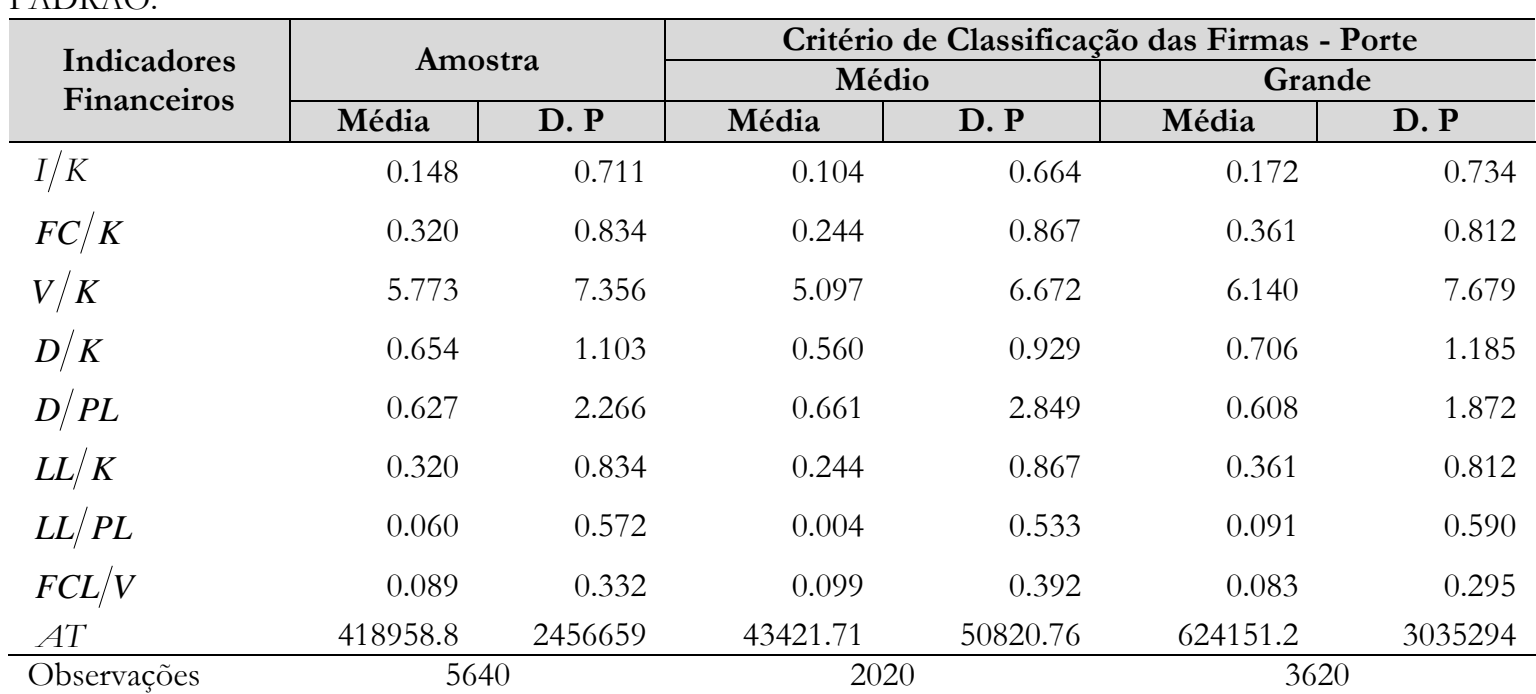

NOTA: Na Tabela 1 foram usadas as seguintes equivalências: $I$ : investimento, $K$ : estoque de capital, $F C$ : fluxo de caixa, FCL : fluxo de caixa livre, $V$ : vendas, $D:$ dívida, $L L$ : lucro líquido, $P L$ : patrimônio líquido, $A T$ : ativo total.

A elevada necessidade de caixa e baixa rentabilidade das firmas de médio porte podem estar sinalizando maior sensibilidade do investimento ao fluxo e maior restrição financeira. Por outro lado, se o fluxo de caixa sinalizar potencial de rentabilidade espera-se que as firmas de grande porte apresentem maior sensibilidade do investimento ao fluxo de caixa e maior restrição financeira.

A Tabela 2 apresenta os parâmetros estimados, considerando os modelos de efeito fixo e aleatório dentro de uma abordagem bayesiana.

Considerando que o modelo de efeito fixo apresentou maior capacidade de predição, conforme o critério da densidade preditiva, a análise dos resultados será baseada nesse modelo. Conforme esperado e de acordo com a maior influência do fluxo de caixa nos gastos com investimentos, os valores estimados para os parâmetros da variável $(F C / K)$ foram significativos na explicação do investimento somente para as firmas de médio porte. A significância do parâmetro do fluxo de caixa aliado a baixa rentabilidade evidencia a presença de restrição financeira para tais firmas, sem estar sujeita a crítica de que o fluxo de caixa esteja atuando como uma proxy para a rentabilidade futura.

O coeficiente da variável $(F I N / K)$ apresentou-se significativo para ambos os grupos, porém com valor maior para as firmas de médio porte, indicando maior dependência de recursos externos dessas firmas. Segundo Devereux e Schiantarelli (1990) firmas de menor porte são menos diversificadas, apresentam maiores riscos e menor capacidade de colaterais, o 
que as colocariam em desvantagem na obtenção de recursos externos. O parâmetro da variável $(V / K)$ mostrou-se significativo para ambos os grupos, evidenciando que variações no estoque de capital fixo respondem às variações na taxa do produto da firma.

TABELA 2 - PARÂMETROS ESTIMADOS POR GRUPOS - VALORES MÉDIOS, DESVIOS PADRÃO, INTERVALO DE CREDIBILIDADE 95\% E ÍNDICES DE CONVERGÊNCIA DE GELMAN E RUBIN.

\begin{tabular}{|c|c|c|c|c|c|c|}
\hline \multirow{2}{*}{ Parâmetros } & \multicolumn{3}{|c|}{ Efeito Fixo } & \multicolumn{3}{|c|}{ Efeito Aleatório } \\
\hline & Médias e D.P. & IC $95 \%$ & $\begin{array}{c}\text { Gelman } \\
\text { Rubin }\end{array}$ & Médias e D.P. & IC $95 \%$ & $\begin{array}{c}\text { Gelman } \\
\text { Rubin }\end{array}$ \\
\hline$\gamma_{1 I}$ & $\begin{array}{r}0.126^{* * *} \\
(0.033)\end{array}$ & {$[0.002 ; 0.126]$} & 1.002 & $\begin{array}{r}-0.075^{* * *} \\
(0.031)\end{array}$ & {$[-0.13 ;-0.013]$} & 1.006 \\
\hline$\gamma_{1 I I}$ & $\begin{array}{r}-0.063 * * * \\
(0.032)\end{array}$ & {$[-0.126 ; 0.001]$} & 0.9998 & $\begin{array}{r}-0.226 * * * \\
(0.031)\end{array}$ & {$[-0.29 ;-0.167]$} & 1.001 \\
\hline$\gamma_{2 I}$ & $\begin{array}{r}-0.024 * * * \\
(0.006)\end{array}$ & {$[-0.04 ;-0.012]$} & 1.001 & $\begin{array}{r}-0.003 \\
(0.006)\end{array}$ & {$[-0.014 ; 0.008]$} & 0.9953 \\
\hline$\gamma_{2 I I}$ & $\begin{array}{r}0.003 \\
(0.005)\end{array}$ & {$[-0.006 ; 0.012]$} & 1.0 & $\begin{array}{r}0.015^{* * *} \\
(0.005)\end{array}$ & {$[0.006 ; 0.024]$} & 1.002 \\
\hline$\beta_{1 I}$ & $\begin{array}{r}0.074^{* * *} \\
(0.014)\end{array}$ & {$[0.047 ; 0.102]$} & 0.9999 & $\begin{array}{r}0.063^{* * *} \\
(0.014)\end{array}$ & {$[0.035 ; 0.091]$} & 0.9962 \\
\hline$\beta_{1 I I}$ & $\begin{array}{r}-0.018 \\
(0.015)\end{array}$ & {$[-0.049 ; 0.011]$} & 1.002 & $\begin{array}{r}-0.023 \\
(0.015)\end{array}$ & {$[-0.054 ; 0.007]$} & 0.9999 \\
\hline$\beta_{2 I}$ & $\begin{array}{r}0.009^{* * *} \\
(0.001)\end{array}$ & {$[0.006 ; 0.012]$} & 1.001 & $\begin{array}{r}0.008^{* * *} \\
(0.002)\end{array}$ & {$[0.005 ; 0.012]$} & 1.008 \\
\hline$\beta_{2 I I}$ & $\begin{array}{r}0.015^{* * *} \\
(0.002)\end{array}$ & {$[0.011 ; 0.019]$} & 0.9999 & $\begin{array}{r}0.014 * * * \\
(0.002)\end{array}$ & {$[0.010 ; 0.018]$} & 1.001 \\
\hline$\beta_{3 I}$ & $\begin{array}{r}0.111^{* * *} \\
(0.009)\end{array}$ & {$[0.093 ; 0.128]$} & 1.004 & $\begin{array}{r}0.111 * * * \\
(0.009)\end{array}$ & {$[0.092 ; 0.130]$} & 1.005 \\
\hline$\beta_{3 I I}$ & $\begin{array}{r}0.093^{* * *} * \\
(0.011)\end{array}$ & {$[0.070 ; 0.115]$} & 1.002 & $\begin{array}{r}0.090^{* * * *} \\
(0.012)\end{array}$ & [0.066; 0.113] & 0.9998 \\
\hline$\sigma_{I}^{2}$ & $\begin{array}{r}0.391 \\
(0.010)\end{array}$ & {$[0.372 ; 0.411]$} & 0.9993 & $\begin{array}{r}0.386 \\
(0.010)\end{array}$ & {$[0.367 ; 0.406]$} & 0.9957 \\
\hline$\sigma_{I I}^{2}$ & $\begin{array}{r}0.319 \\
(0.011)\end{array}$ & {$[0.299 ; 0.342]$} & 0.9999 & $\begin{array}{r}0.313 \\
(0.011) \\
\end{array}$ & {$[0.293 ; 0.335]$} & 0.9999 \\
\hline
\end{tabular}

NOTA: ${ }^{a}$ Os desvios padrão estão entre parênteses. ${ }^{b}$ Os subscritos I e II indicam os grupos de firmas de médio e grande porte, respectivamente. ${ }^{c} \mathrm{O}$ símbolo $* * *$ indica probabilidade a posteriori igual 0.99 do verdadeiro valor do parâmetro pertencer ao intervalo de credibilidade obtido.

O resultado encontrado deixa claro que não basta que o parâmetro do fluxo de caixa seja significativo para considerar uma firma restrita financeiramente, já que o fluxo de caixa poderia estar sinalizando oportunidades futuras de investimento e expectativas de rentabilidade.

\section{CONCLUSÃO}

Neste estudo buscou-se analisar a presença de restrição financeira no comportamento do investimento de 564 firmas brasileiras no período de 1997-2006, controladas por porte e considerando uma abordagem bayesiana. O critério da densidade preditiva indicou que o modelo de efeito fixo foi preferível ao modelo de efeito aleatório. 
O principal resultado econômico deste estudo indica que as firmas de médio porte sofrem restrições financeiras nas decisões de investimento. Ao classificar as firmas por porte, a maior sensibilidade do investimento ao fluxo de caixa é apresentada para as firmas de médio porte, as quais mostraram piores indicadores financeiros. Esta classificação parece indicar uma forma de controlar os efeitos das restrições financeiras de outros fatores, já que o fluxo de caixa não estaria sujeito à crítica de atuar como proxy para a rentabilidade futura. Como apontado no seminal estudo de Stiglitz e Weiss (1981), a propensão de uma empresa estar sujeita a restrição de crédito não é neutra em relação ao tamanho da empresa. A presença de restrição para as firmas menores estaria indicando menor disponibilidade de colaterais como garantias, serem menos diversificadas, além de dispor de pouca informação sobre a capacidade gerencial e oportunidades de investimento. Por outro lado, as firmas de grande porte não se apresentaram como restritas financeiramente, devido sua maior facilidade em obter recursos externos e melhores condições financeiras. 


\section{REFERÊNCIAS}

CLEARY, S. International corporate investment and the relationships between financial constraint measures. Journal of Banking \& Finance, v. 30, p. 1559-1580, 2006.

DEVEREUX, M.; SCHIANTARELLI, F. Investment, Financial Factors, and Cash Flow: Evidence from U.K Panel Data. In: HUBBARD, R.G. Asymmetric Information, Corporate Finance, and Investment. Chicago: Chicago University Press, 1990.

FAZZARI, S. M.; HUBBARD, G.; PETERSEN, B. Financing Constraints and Corporate Investment. Brookings Papers on Economic Activity, v. 1, p. 141-95, 1988.

GELMAN, A.; RUBIN, D. Inference form Iterative Simulation Using Multiple Sequence. Statistical Science, v. 7, n. 4, p. 457-472, 1992.

GELFAND, A.; DEY, K. D. Bayesian Model Choice: Asymptotics and Exacts Calculations. Journal of the Royal Statistician Society, Series B, v. 56, n. 3, p. 501-514, 1993.

GILCHRIST, S.; HIMMELBERG, C. P. Evidence on the role of cash flow for investment. Journal of Monetary Economics, v. 36, p. 541-572, 1995.

HSIAO, C.; TAHMISCIOGLU, A. K. A Panel Analysis of Liquidity Constraints and Firm Investment. Journal of the Statistical Association, v. 92, n. 438, p. 455-465, 1997.

KALATZIS, A. E. G.; AZZONI, C. R. Investment decisions in troubled times: A Bayesian approach applied to Brazilian firms. International Journal of Production Economics, p. 595-606, 2009.

KAPLAN, S.; ZINGALES, L. Do Investment-cash flow sensitivities provide useful measures of financing constraints? Quarterly Journal of Economics, v. 122, p. 169-215, 1997.

SCHALLER, H. Asymmetric information, liquidity constraints, and Canadian investment. Canadian Journal of Economics, v. 26, n. 3-4, p. 552-74, 1993.

SPIEGELHALTER, D. J.; THOMAS, A.; BEST, N. G.; GILKS, W. R. Winbugs: Bayesian Inference using Gibbs Sampling. MRC. Biostatistics Unit, Cambridge, 1995.

STIGLITZ, J.; WEISS, A. Credit Rationing in Markets with Imperfect Information. American Economic Review v. 71, p. 393-410, 1981. 
